\section{CTCF-mediated topological boundaries during development foster appropriate gene regulation}

\author{
Varun Narendra, ${ }^{1,2}$ Milica Bulajić, ${ }^{3}$ Job Dekker, ${ }^{1,4}$
} Esteban O. Mazzoni, ${ }^{3}$ and Danny Reinberg ${ }^{1,2}$

${ }^{1}$ Howard Hughes Medical Institute, New York, New York 10016, USA; ${ }^{2}$ Department of Biochemistry and Molecular

Pharmacology, New York University School of Medicine, New York, New York 10016, USA; ${ }^{3}$ Department of Biology, New York University, New York, New York 10003, USA; ${ }^{4}$ Program in Systems Biology, Department of Biochemistry and Molecular Pharmacology, University of Massachusetts Medical School, Worcester, Massachusetts 01605, USA

The genome is organized into repeating topologically associated domains (TADs), each of which is spatially isolated from its neighbor by poorly understood boundary elements thought to be conserved across cell types. Here, we show that deletion of CTCF (CCCTC-binding factor)-binding sites at TAD and sub-TAD topological boundaries that form within the $\operatorname{Hox} A$ and $\operatorname{Hox} C$ clusters during differentiation not only disturbs local chromatin domain organization and regulatory interactions but also results in homeotic transformations typical of Hox gene misregulation. Moreover, our data suggest that CTCF-dependent boundary function can be modulated by competing forces, such as the self-assembly of polycomb domains within the nucleus. Therefore, CTCF boundaries are not merely static structural components of the genome but instead are locally dynamic regulatory structures that control gene expression during development.

Supplemental material is available for this article.

Received July 31, 2016; revised version accepted December 12, 2016.

Precise control of gene expression ensures correct embryonic development. Gene regulation is established by a nested organization of the genome in space, ranging from dynamic local DNA loops to large-scale topologically associated domains (TADs) (Dixon et al. 2012; Nora et al. 2012). Within this hierarchical organization, conserved TAD and sub-TAD structures frame the majority of enhancer-promoter interactions (Dekker et al. 2013; Tang et al. 2015; Dekker and Mirny 2016). The CCCTCbinding factor (CTCF) regulates each nested level of genomic organization (Guo et al. 2015; Hnisz et al. 2016), from local enhancer-promoter looping events within the protocadherin clusters to broader genomic neighborhoods and TADs (Guo et al. 2012; Rao et al. 2014).

[Keywords: CTCF; chromatin and epigenetics; Hox gene regulation; Polycomb/Trithorax; TADs; chromosomal conformation] Corresponding authors: danny.reinberg@nyumc.org, eom204@nyu.edu Article is online at http://www.genesdev.org/cgi/doi/10.1101/gad.288324. 116.
Although a macroscopic megabase-scale view of TADs suggests that their genomic position is robustly conserved across cell types, TAD and sub-TAD boundaries can be rather dynamic at a local kilobase level such that they are repositioned in distinct cell types during development to orchestrate local cell type-specific genomic contacts. Although sub-TADs have been shown specifically to be dynamic during differentiation, regulatory mechanisms underlying these dynamics remain poorly understood. Here, we used the model system of motor neuron (MN) differentiation to study CTCF-dependent TAD and subTAD boundaries that fall within the Hox clusters and determine how they play cell type-specific roles in controlling chromatin interactions for individual Hox genes and Hox expression during development. By using 5C (chromosome conformation capture carbon copy) to analyze the allocation of Hox genes into different topological domains during $\mathrm{MN}$ differentiation, we demonstrate that $\mathrm{TAD}$ and sub-TAD boundaries are not rigid in their genomic position. Rather, they can be flexible in the extent to which they block or permit cell type-specific chromatin interactions and gene expression. Furthermore, by deleting CTCF-binding sites within the Hox clusters, we show that TAD boundaries are formed by the long-range self-assembly of CTCF molecules in space. We also provide data suggesting that their cell type specificity may arise from rearrangements in local chromatin structure that allow for different patterns and insulation capacity of long-range CTCF interactions. Genetic disruption of these mechanisms manifests as homeotic transformations typical of Hox gene misregulation, including defects in axial skeletal patterning in vivo and MN subtype specification in vitro.

\section{Results and Discussion}

In vertebrates, paralogous families of Hox genes are densely distributed across four clusters $\operatorname{Hox} A, \operatorname{Hox} B, \operatorname{Hox} C$, and $\operatorname{Hox} D)$, the expression of which is controlled by shortand long-range DNA looping (Duboule 2007; Maeda and Karch 2009; Montavon et al. 2011; Lonfat et al. 2014). Thus, cluster positioning relative to TAD structure has the potential to reduce or facilitate the association of long-distance regulatory regions and Hox genes. While both the HoxA and HoxC clusters are able to fold within local sub-TAD conformations, they do not occupy a consistent position within the larger TAD structure. Analysis of available Hi-C (chromosome capture followed by highthroughput sequencing) data shows that, while the $\operatorname{HoxC}$ cluster lies entirely within a TAD, the HoxA cluster intersects a TAD boundary in embryonic stem cells (ESCs) (Supplemental Fig. S1A,B; Dixon et al. 2012; Lonfat and Duboule 2015). However, the precise region of this intersection remains unclear given the limited resolution of Hi-C. Thus, we performed comprehensive $5 \mathrm{C}$ across a 4$\mathrm{Mb}$ region spanning the $\operatorname{Hox} A$ cluster to define its

(C) 2016 Narendra et al. This article is distributed exclusively by Cold Spring Harbor Laboratory Press for the first six months after the full-issue publication date (see http://genesdev.cshlp.org/site/misc/terms.xhtml). After six months, it is available under a Creative Commons License (Attribution-NonCommercial 4.0 International), as described at http://creativecommons.org/licenses/by-nc/4.0/. 
location within the context of TADs (Fig. 1A). We did so in MNs derived from wild-type mouse ESCs, as we had already developed 4C (circularized chromatin conformation capture) maps of the local topology and chromatin structure of the Hox loci for these cells (Mazzoni et al. 2013; Narendra et al. 2015). TAD boundaries were determined by calculating an "insulation score" at each position along the 4-Mb scan, which reflects the aggregate of interactions (within an $~ 160-k b$ window) occurring across each genomic position (Crane et al. 2015). Local minima of the insulation profile denote areas of high insulation or TAD boundaries. Visual inspection of the 5C interaction map and the associated insulation profile reveals at least 10 TAD boundaries, with possibly weaker boundaries present as well (Fig. 1A,B). These data are highly reproducible, as an independent biological 5C replicate reveals the equivalent chromosomal structure (Supplemental Fig. S2A,B). Interestingly, in MNs, the boundary between two of these TADs intersects the HoxA cluster near the CTCF motif located between Hoxa5 and Hoxa6 (a5|6), aligning exactly with the boundary between adjacent active and repressed chromatin domains that is formed within the cluster during $\mathrm{MN}$ differentiation (Fig. 1C; Supplemental Fig. S2C). This is in contrast to ESCs, in which the TAD boundary localizes roughly to a10|11 (Fig. 1B,C; Supplemental Fig. S2B,C), suggesting that the precise positioning of TAD boundaries is locally dynamic during differentiation. Each of these boundaries engages in long-range looping interactions that are present in both ESCs and MNs, consistent with the fact that both cell types have the same CTCF-binding profile. These are identified as corner peaks within the $5 \mathrm{C}$ heat map
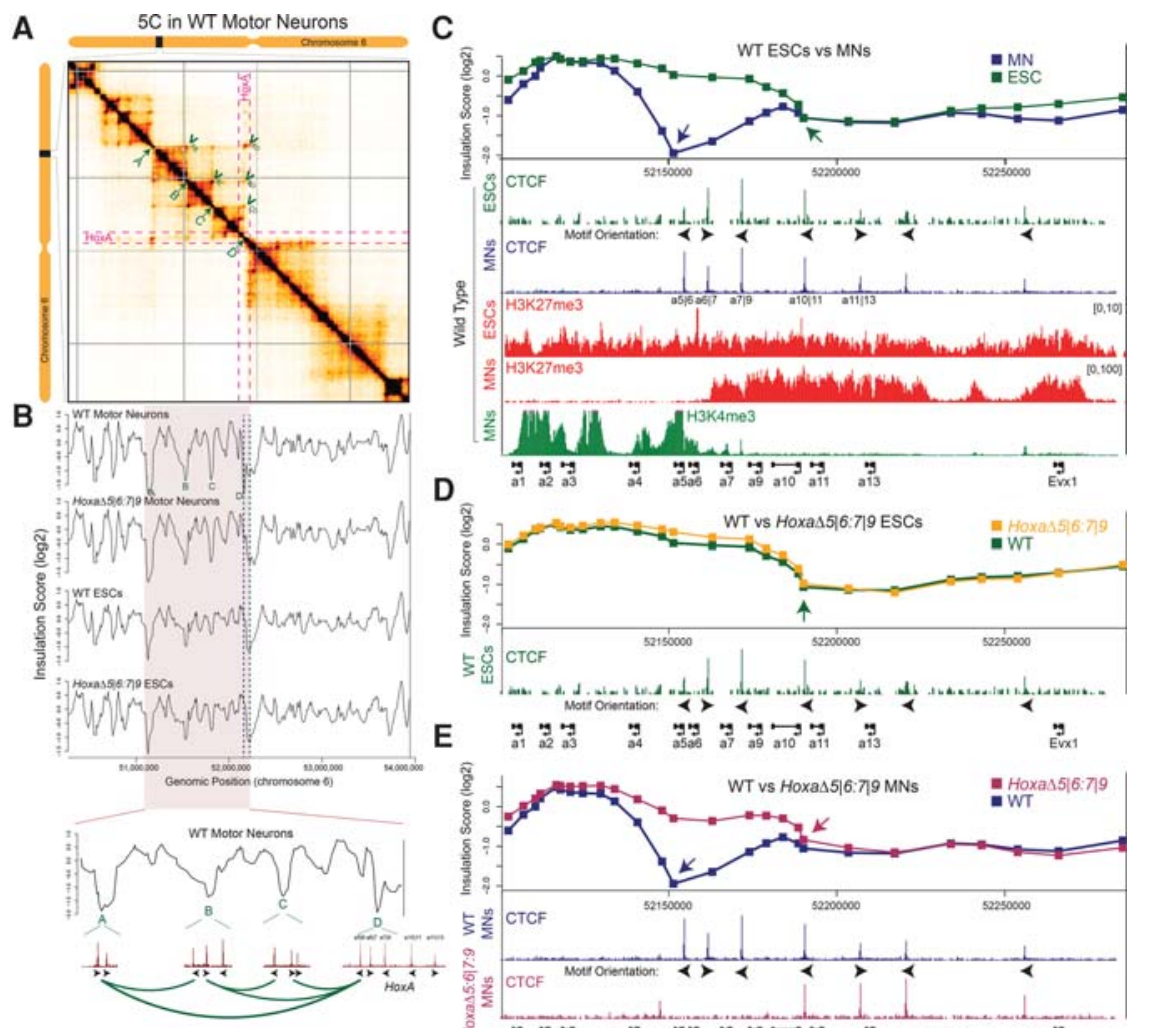

is
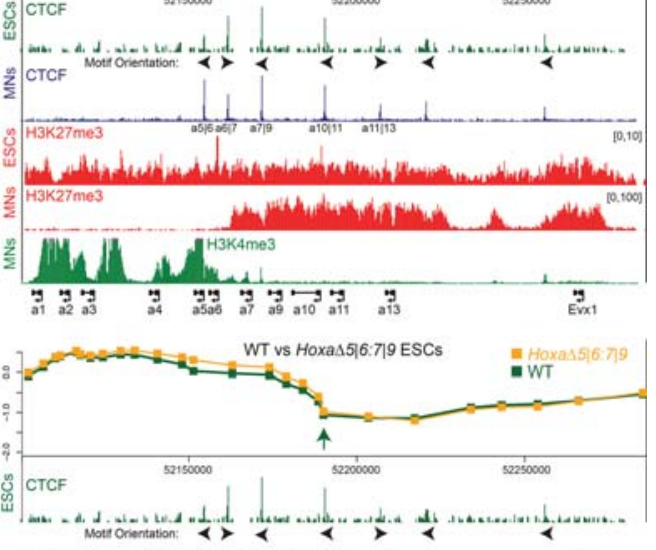

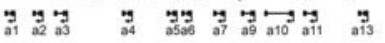

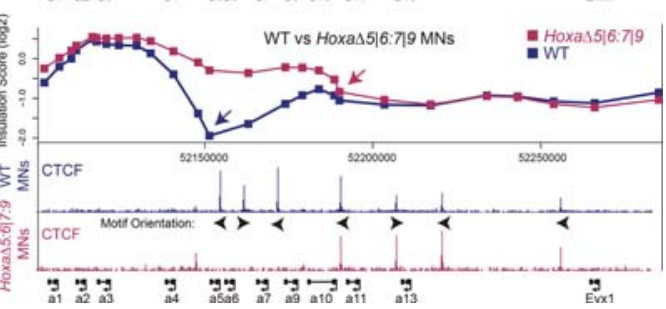

Figure 1. CTCF underlies the dynamic TAD boundaries during differentiation. $(A)$ The 5C chromatin interaction heat map for wild-type $\mathrm{MN}$ cells depicts the interaction frequency between restriction fragments across a $4-\mathrm{Mb}$ region surrounding the Hox A cluster (data were binned in 15-kb windows; step size $5 \mathrm{~kb}$; the median is shown). Darker colors represent increasing interaction frequency. Gray lines represent missing data. The dotted magenta line bounds the extent of the HoxA cluster within the heat map. Topological boundaries within the region of the genome that form loops to interact with the a5|6 position are labeled in green. $(B)$ Insulation profiles $\left(\log _{2}\right)$ of wild-type and Hoxa $45|6: 7| 9$ ESCs and MNs. The dotted blue line indicates the TAD boundary present in wild-type MNs at the a5 $\mid 6$ position. The dotted green line indicates the TAD boundary present in Hoxa $\triangle 5|6: 7| 9$ MNs near the a10|11 position, which also matches the boundary found in wild-type and Hoxa $\Delta 5|6: 7| 9$ ESCs. The genomic region that interacts with the $\operatorname{Hox} A$ cluster is highlighted, and the associated insulation profile is magnified below. CTCFbinding profiles within TAD boundaries are displayed, along with motif orientation. Convergent motifs point toward each other, and the corner peaks identified in the $5 \mathrm{C}$ heat map are represented by green loops. (C-E) ChIP-seq (chromatin immunoprecipitation [ChIP] sequencing) tracks for the indicated proteins and histone modifications across the Hox $A$ cluster in the indicated cell types and genetic backgrounds. ChIP-seq data were obtained from GSE60232 (Narendra et al. 2015). Insulation scores $\left(\log _{2}\right)$, as calculated in $B$, are depicted above. Blue and green arrows point to TAD boundary positions within the HoxA cluster, which lie at the minima of the insulation score. The relative orientations of the CTCF motifs within the HoxA cluster are displayed.

(Fig. 1A; Supplemental Fig. S2A, green arrows). Of note, CTCF-binding events not only are found precisely at these long-range interaction sites but are positioned in a convergent orientation with respect to a5|6, as required for a loop extrusion model for their interaction (Fig. 1B, bottom; Supplemental Fig. S2B). Although present, the long-range interactions with a5|6 are altered and weaker in undifferentiated ESCs (Supplemental Fig. S3, blue arrows). Meanwhile, the a5|6 CTCF site allows for significant interactions to occur across it in ESCs, unlike the case in MNs (Supplemental Figs. S3, S4A; Narendra et al. 2015). Here, the pattern and insulation strength of CTCF-mediated loops are cell typespecific despite the fact that CTCF binding within the HoxA cluster is unchanged between ESCs and MNs (Fig. 1C). These findings suggest that although CTCF is capable of making all of the requisite spatial contacts needed to form a TAD boundary in ESCs, its capacity for local insulation on the $\sim 100-\mathrm{kb}$ scale is quantitatively different in ESCs and MNs. One possibility is that this is related to the presence of polycomb complex binding on either side of the a5|6 position in ESCs (Fig. 1C). We suggest that the property of polycomb to compact chromatin and self-aggregate into spatial hubs within the nucleus is sufficient to override CTCF-dependent insulation. Taken together, CTCF seems to landmark potential boundary regions of the cluster in both ESCs and MNs and engages in long-range interactions with other CTCF sites in both cell lines. However, the boundary activity of any given CTCF site is tunable, as demonstrated by the increased TAD boundary activity in MNs. As cells differentiate and as the local chromatin and regulatory landscape at CTCF boundaries changes, the range and stability of long-range looping events 
with other CTCF sites can be very dynamic between cell types (Vieux-Rochas et al. 2015).

To formally demonstrate that CTCF-mediated looping events regulate $\mathrm{TAD}$ reorganization during development, we performed $5 \mathrm{C}$ before and after $\mathrm{MN}$ differentiation of ESCs in which the CTCF-binding sites between Hoxa5 and Hoxa6 (Hoxa $\Delta 5 \mid 6)$ and Hoxa7 and Hoxa9 (Hoxa $47 \mid 9)$ were both mutated (Hoxa $5|6: 7| 9)$ (Supplemental Fig. S5A). This causes a loss of the a6|7-binding event as well; thus, these cells lack three consecutive CTCF-binding events at the HoxA cluster (Fig. 1E). Although the loss of CTCF binding in ESCs results in a slightly increased interaction frequency within the rostral segment of the HoxA cluster and a mild increase in interactions across the sites over larger distances (Supplemental Fig. S4B), the TAD boundary position at a10|11 remains unchanged (Fig. 1D; Supplemental Fig. 2D). Upon differentiation into MNs, however, CTCF binding at a5 $|6, \mathrm{a} 6| 7$, and a $7 \mid 9$ is required for the precise positioning of a strong TAD boundary. While the remaining TAD boundaries are intact, the single TAD boundary between Hoxa5 and Hoxa6 is now absent in Hoxa $\triangle 5|6: 7| 9$ MNs (Fig. 1E; Supplemental Fig. 2E). Instead, the rostral TAD extends to the new topological boundary roughly between Hoxa10 and Hoxa11, in accordance with the aberrant transcriptional activation of Hoxa7, Hoxa9, and Hoxa10 observed previously in these cells (Narendra et al. 2015). Indeed, the orientation of the a10|11 CTCF-binding motif is equivalent to that of a5|6, allowing it to function as a new TAD anchor point. Together these results show that CTCF-binding sites a5 $\mid 6$, a6 6 , and a $7 \mid 9$ display cell type-specific quantitative differences in boundary activity: A weak boundary is formed in ESCs, while the same sites form a strong boundary in MNs.

Showing that CTCF mediates long-distance interactions in both cell types, we found that, in both Hoxa $\Delta 5$ 6:7|9 ESCs and MNs, several specific looping interactions detected as corner peaks that engage with CTCF at a5 6 are lost (Supplemental Fig. S3, blue arrows). To quantitatively demonstrate this, we calculate the change in $5 \mathrm{C}$ interaction frequencies between wild-type and Hoxa $45|6: 7| 9$ ESCs and MNs. In both cell types, we observed vertical and horizontal blue lines that project onto the a5 $|6: a 7| 9$ region. This linear pattern of differential interaction frequency along the boundary of the HoxA-intersecting TAD signifies a loss of CTCF-mediated looping events that may have formed via a loop extrusion model (Supplemental Fig. S4). Interestingly, the Hoxa $\Delta 5|6: 7| 9$ deletion has a more profound effect on CTCF-mediated looping in MNs than in ESCs (Supplemental Fig. S4), in agreement with the model that HoxA CTCF sites are commissioned in $\mathrm{MNs}$ to engage in long-range interactions and less so in ESCs, where these CTCF-binding events are buried within a compact polycomb domain. Further underscoring the importance of CTCF to the structural integrity of TADs, we observed substantially diminished insulation across the HoxA TAD boundary in Hoxa $45|6: 7| 9$ cells (Supplemental Fig. S4, rectangular regions of increased [red] interaction frequency). Combined, these results reveal that the a5|6:a7|9 CTCF sites play a complex role in higher-order chromatin organization by looping to other CTCF sites in both cell types but with different patterns and frequencies and by displaying cell type-specific quantitative differences in boundary strength.

The dynamic nature of TAD boundaries across the HoxA cluster suggests that these structures play a critical role in regulating developmental gene expression. Hox regulation of $\mathrm{MN}$ subtype identity presents a natural system in which to test the developmental relevance of TAD boundary dynamics. The generation of distinct MN subtype identities along the spinal cord during development necessitates precise transcription profiles from the Hox clusters, with genes from the HoxC cluster dominating this patterning (Beuchle et al. 2001). Ectopic expression of a single Hox gene, Hoxc6, within the thoracic spinal cord is sufficient to induce an ectopic limb-innervating lateral motor column (LMC) fate to developing MNs by inducing the expression of the transcription factor Foxp1 (Dasen et al. 2008; Rousso et al. 2008). Similar to the HoxA setting, we previously identified a CTCF-binding site between Hoxc5 and Hoxc6 (c5|6) that, upon deletion, results in ectopic Hoxc6 expression during differentiation (Supplemental Fig. S5B; Narendra et al. 2015). Although a TAD boundary has not been assigned in the literature to intersect the Hox C cluster (Supplemental Fig. S1B), the remarkably similar behavior of the Hoxa $\Delta 5 \mid 6$ and Hoxc $\Delta 5 \mid 6$ mutants argues that these boundaries function quite similarly. By differentiating Hoxa $\Delta 5 \mid 6$ and Hoxc $\Delta 5 \mid 6$ cells (which harbor single 5|6 CTCF deletions in the HoxA and Hox $C$ clusters, respectively), we could then test whether misallocation of HoxA or HoxC genes across CTCFdelimited topological boundaries would alter transcriptional output to an extent sufficient to encroach on $\mathrm{MN}$ subtype identity during in vitro differentiation.

The cervical-to-brachial transition along the rostrocaudal spinal cord axis is controlled by the expression of Hox6 paralogs that instruct a fraction of brachial MNs to become limb-innervating LMC MNs expressing Raldh2 and Foxp1 (Dasen et al. 2008; Rousso et al. 2008). To test whether aberrant Hox6 expression in the Hoxa $\Delta 5 \mid 6$ and Hoxc $\Delta 5 \mid 6$ cells results in MN caudalization, we measured the mRNA levels of Raldh2 and Foxp1. Under the conditions used to differentiate ESCs in vitro, all mutant and wild-type cell lines differentiate equally well into MNs, with $\sim 40 \%$ of cells expressing the MN marker Is $11 / 2$ (wild type: $43 \%$; Hoxa $\Delta 5 \mid 6: 42 \%$; and $H o x c \Delta 5 \mid 6: 43 \%$ of total cells). Wild-type cells activate rostral Hox gene expression during in vitro differentiation with retinoic acid, favoring non-LMC cervical MN fates. However, the Hoxc $\Delta 5 \mid 6$ mutation induces the expression of both Raldh2 and Foxp1 (Fig. 2A). On the other hand, the Hoxa $\Delta 5 \mid 6$ mutation fails to induce Raldh2 or Foxp1 above the wild-type control (Fig. 2A). To confirm that the Hoxc $\Delta 5 \mid 6$-specific phenotype is due to an increase in the percentage of MNs that have achieved a caudal identity, we quantified across all cell lines the fraction of Is11/2positive MNs that induce Foxp1. While only $11.9 \% \pm$ $0.3 \%$ of wild-type and $12.6 \% \pm 1.5 \%$ of Hoxa $\Delta 5 \mid 6$ Is $11 / 2$ positive MNs express Foxp1, the fraction of Foxp1-expressing MNs is significantly increased to $22.4 \% \pm 1.2 \%$ in the Hoxc $\Delta 5 \mid 6$ mutant (Fig. 2B,C). Arguing that this phenotype is secondary to Hoxc6 activation, transgenic overexpression of Hoxc6 on a wild-type background (inducible Hoxc6 [iHoxc6] cell line) during differentiation phenocopies the Hoxc $\Delta 5 \mid 6$ mutant by up-regulating Raldh2 and Foxp1 and increasing the fraction of Foxp1-expressing MNs to $26.0 \% \pm 1.0 \%$ (Fig. $2 \mathrm{~A}-\mathrm{C}$ ). The difference between the CTCF mutant lines in the HoxA and HoxC cluster is consistent with published data suggesting that Hoxc6, rather than Hoxa6, specifically regulates Foxp1 expression in MNs (Lacombe et al. 2013). In summary, the deletion of a CTCF-dependent local topological boundary within the $\operatorname{Hox} C$ cluster disrupts its organization such 
A
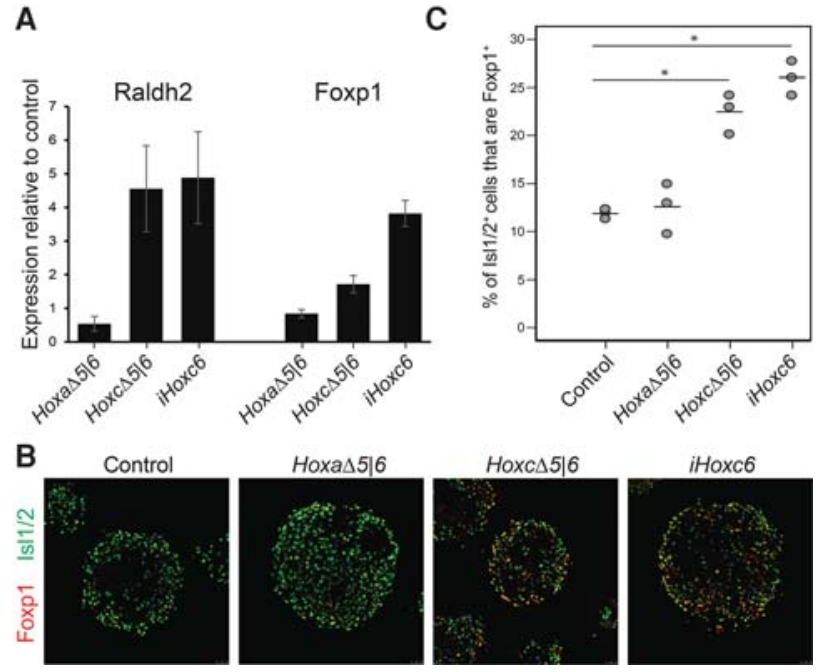

Hoxa $\Delta 5 \mid 6$
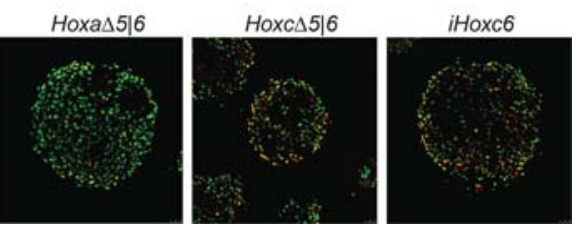

Figure 2. CTCF boundaries regulate the positional identity of MNs. (A) RT-qPCR analysis of expression levels of LMC markers (Raldh2 and Foxp1) in Hoxa $\Delta 5|6, H o x c \Delta 5| 6$ as well as Hoxc6-overexpressing (iHoxc6) cell lines relative to the wild-type control. Expression levels from five different biological replicates were normalized to the expression levels of a generic MN marker (Chat) to account for differences in MN numbers between different lines. $(B)$ Representatives of differentiated embryoid bodies from control, Hoxa $\Delta 5 \mid 6$, Hoxc $\Delta 5 \mid$ 6, and Hoxc6-overexpressing (iHoxc6) cell lines stained by the MN marker Isl1/2 and the LMC transcription factor Foxp1. (C) Quantification of Foxp1 expression in Isl1/2-expressing MNs in three independent differentiations \pm SEM. $\left(^{*}\right) P<0.005$, one-tailed unpaired $t$-test.

that ectopic Hoxc6 expression induces a homeotic transformation-like phenotype even under very restricted in vitro differentiation conditions.

While the topological organization of the Hox clusters plays a critical role in the establishment of positional identity in ESC-derived MNs, whether this principle generalizes to the wide array of developmental processes that occur in vivo is unknown. Nested Hox expression patterns the mesoderm-derived axial skeleton along the rostro-caudal axis (Garcia-Gasca and Spyropoulos 2000; McIntyre et al. 2007). Thus, we tested the CTCF-demarcated TAD boundaries within the HoxA and HoxC clusters for their input into skeletal patterning during embryonic development. To generate precise deletions of CTCF elements within the Hox clusters, we first performed zygotic injections of Cas9 mRNA and guide RNAs (gRNAs) targeting both the a5 6 and a $7 \mid 9$ motifs. Implantation of the resulting embryos into a pseudopregnant mouse produced a pup harboring 7-base-pair (bp) and 2-bp deletions in cis within the a5 6 and a7 $\mid 9$ motifs, respectively $($ Hoxa $45|6: 7| 9)$ (Fig. 3A). Separately, zygotes were injected with a gRNA targeting the c5|6 motif, resulting in

A

B sented in blue. two heterozygous genotypes: one harboring a 76-bp deletion across the motif and another harboring a 12-bp deletion $\left(H_{0 x c} 45 \mid 6\right)$ (Fig. 3A). Upon adulthood, these heterozygous mice were then crossed, producing wild-type/ wild-type, 12-bp/wild-type heterozygous, 76-bp/wildtype heterozygous, 12-bp/76-bp homozygous, 12-bp/12bp homozygous, or 76-bp/76-bp homozygous offspring. Postnatal day 0.5 (P0.5) neonates from this cross were dissected and stained with Alcian blue and Alizarin red to reveal their underlying axial skeletal distribution. Wild-type mice have seven cervical, 13 thoracic, six lumbar, and four sacral vertebrae, and transitions between these segments are regulated by Hox genes. In vivo, every analyzed Hoxc $\Delta 5 \mid 6$ heterozygous $\left(\mathrm{HoxC}^{+/ \Delta}\right)$ and homozygous $\left(\operatorname{HoxC}^{\Delta / \Delta}\right)$ mouse exhibits a C7-to-T1 posterior homeotic transformation at the cervicothoracic boundary within the axial skeleton (Fig. 3B,C). This finding demonstrates a clear developmental defect associated with the disruption of a topological boundary. In these mice, an extra rib aberrantly protrudes from the $\mathrm{C} 7$ position and forms a proper costochondral joint with cartilage arising from the sternum. Importantly, the total number of vertebrae is unchanged relative to wild type, consistent with the length of the rostro-caudal axis being normal. Similarly, the interTAD Hoxa $\Delta 5|6: 7| 9$ mutation results in a skeletal patterning defect, albeit at the thoracolumbar transition. Here, proper ribs do not protrude from the T13 position. This phenotype occurs with variable expressivity, presenting as unilateral or bilateral agenesis or shortened ribs, and occurs with $76 \%$ penetrance in homozygous mice $\left(\operatorname{Hox}^{\Delta / \Delta}\right)$ (Fig. 3B,C). This is opposite to that observed in Hoxa9 knockout mice, in which an extra rib protrudes from the L1 position, arguing that it is most likely the result of
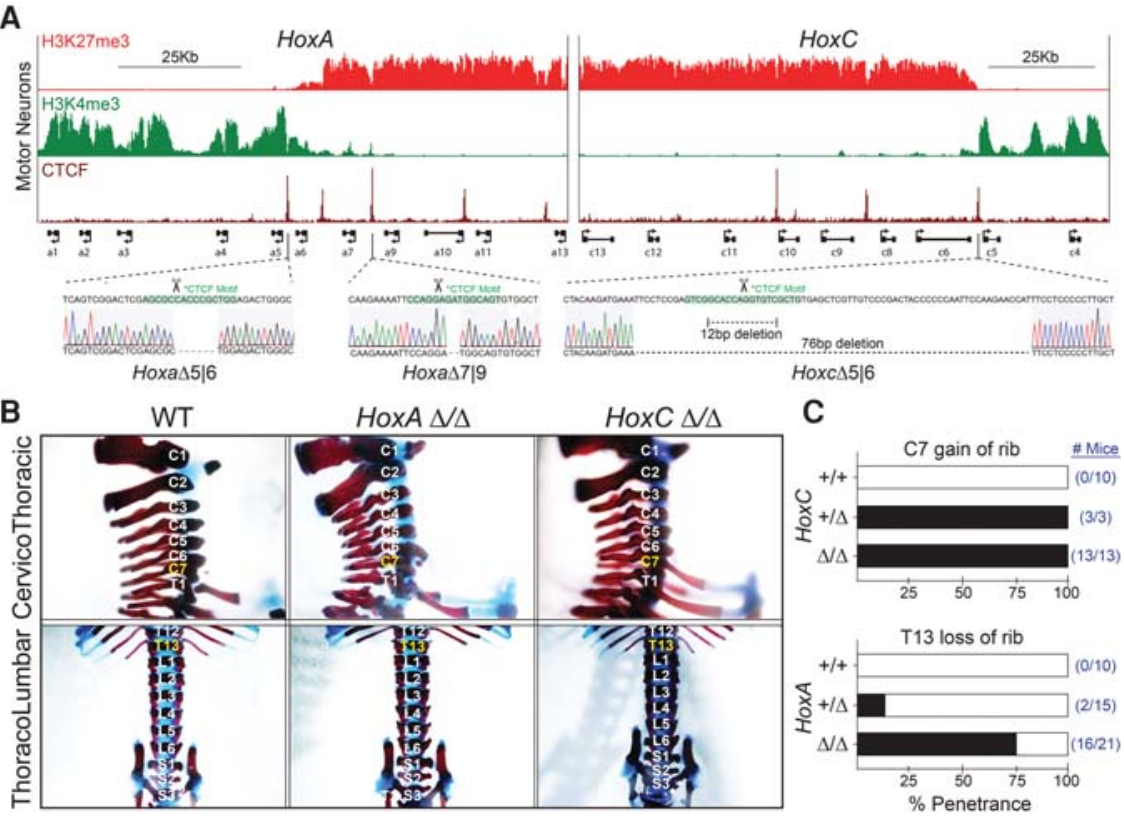

C

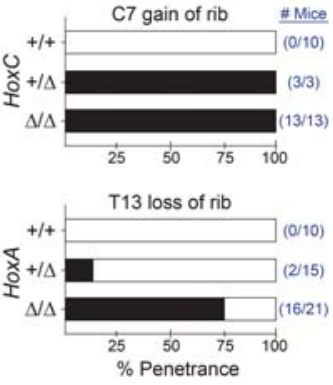

Figure 3. CTCF-delimited TAD boundaries correspond to boundaries in axial-skeletal patterning in vivo. (A) ChIP-seq tracks for the indicated proteins across the HoxA (left) and HoxC (right) clusters and sequencing chromatograms of CTCF-binding site deletions (bottom). ChIP-seq is from GSE60232 (Narendra et al. 2015). (B) Representative Alcian blue-Alizarin red stain of P0.5 pups in Hoxa $\Delta 5|6: 7| 9$ and Hoxc $\Delta 5 \mid 6$ homozygous animals $\left(H o x A^{\Delta / \Delta}\right.$ and $H o x C^{\Delta / \Delta}$, respectively). (C) Phenotypic penetrance in the indicated genetic backgrounds. Raw numbers are pre- 
altered rostral spatiotemporal activation of Hoxa9 in the setting of CTCF ablation. These findings agree with lossof-function studies that have shown that Hoxc6 and Hoxa9 function at the cervicothoracic and thoracolumbar transitions, respectively (Garcia-Gasca and Spyropoulos 2000; Dasen et al. 2003; McIntyre et al. 2007). Thus, changing the accessibility of Hox genes to regulatory elements during embryonic development by deleting CTCF sites located both within and at the boundary of topological domains results in a profound misregulation of Hox gene expression and leads to homeotic transformations.

Mutations in CTCF-binding sites alter not only the three-dimensional structure of the genome but also the regulation of gene expression (Mazzoni et al. 2013; Guo et al. 2015; Narendra et al. 2015). Studies on the developmental relevance of TADs in vivo have used multikilobase to megabase length deletions that disrupt TAD organization (Lupiáñez et al. 2015). The results presented here demonstrate that precise CTCF-binding elements lay the molecular framework on which TAD and sub-TAD structures form. Genetic perturbation of this framework has profound effects on gene regulation and development even when studied within the context of a highly redundant system like Hox gene-dependent patterning. We show that deletion of a single CTCF-binding event within the $\operatorname{Hox} C$ cluster is sufficient to drive differentiating MNs toward an altered caudal cell fate. Importantly, this mode of regulation extends to the complex setting of embryonic development; CTCF-binding site deletions at TAD and sub-TAD boundaries within the $\operatorname{Hox} A$ or $\operatorname{Hox} C$ clusters, respectively, induce homeotic transformations in mice, substantiating the importance of these topological regulatory elements in developmental gene expression.

Relocating a gene or genomic segment from one side of a topological boundary to the other dramatically changes its regulatory associations and thus transcriptional output (Hnisz et al. 2016; Neijts et al. 2016). We propose that a subset of CTCF elements is poised to function as TAD boundaries in all cell types, yet their capacity for insulation can be modulated in a cell type-specific manner. This flexibility-as evident by the example of Hox clusters-can facilitate the rearranging regulatory landscapes that evolve during development. CTCF binding is stable along the Hox clusters during differentiation, yet interior CTCF sites acquire a TAD boundary function by both forming new long-range looping interactions and strengthening existing contacts, specifically as they differentiate into MNs. We propose that this cell type-specific modulation in CTCF-mediated boundary activity appears to be responsive to the local chromatin environment. In undifferentiated cells, the insulating capacity of CTCFmediated loops is overridden by self-assembling polycomb domains. Upon differentiation, Hox clusters are compartmentalized into active and repressed domains, allowing a CTCF-mediated looping framework to shape regulatory contacts. Such regulation draws attention to the remarkable and dynamic customization of genome partitioning inherent in the timely progression of gene regulation during normal development.

\section{Materials and methods}

\section{Cell culture and MN differentiation}

ESCs were grown in standard ESC medium containing Lif, $1 \mu \mathrm{M}$ MEK1/2 inhibitor (PD0325901), and $3 \mu \mathrm{M}$ GSK3 inhibitor (CHIR99021). ESC differ- entiation was described previously (Wichterle et al. 2002). See the Supplemental Material for a detailed protocol.

\section{Expression analysis}

RNA was purified from cells with TRIzol (Invitrogen). RT-PCR was performed (Invitrogen) in replicate on 300 ng of total RNA followed by qPCR using a SYBR Green master mix (Thermo Scientific).

\section{Preparation of 5C libraries}

5C was performed as described by Dostie and Dekker (2007). Two biological replicates were processed separately. See the Supplemental Material for a detailed protocol.

\section{CRISPR zygotic injection}

HoxA and HoxC mutant mice were generated by zygotic injection (Yang et al. 2014). Detailed methods are in the Supplemental Material.

\section{Alcian blue-Alizarin red staining}

P0.5 neonates were dissected by removing the skin and organs completely. Specimens were fixed for $24 \mathrm{~h}$ in $100 \%$ ethanol with rocking at room temperature. Ethanol was replaced with Alcian blue stain $10.03 \%$ Alcian blue, $80 \%$ ethanol, $20 \%$ acetic acid) for $24 \mathrm{~h}$ with rocking at room temperature. Embryos were fixed again for $6 \mathrm{~h}$ in ethanol and transferred to $2 \% \mathrm{KOH}$ solution for 12-24 h. Any residual skin or tissue was removed at this point. The specimen was subsequently stained with Alizarin red solution (0.03\% Alizarin red, $1 \% \mathrm{KOH}$ in water) for $12-24 \mathrm{~h}$. The skeleton was then further cleared in $1 \% \mathrm{KOH} / 20 \%$ glycerol and then $1: 1$ glycerol: ethanol. For long-term storage, embryos were transferred to 4:1 glycerol: ethanol.

\section{Immunocytochemistry}

Embryoid bodies and MNs were fixed with $4 \%(\mathrm{v} / \mathrm{v})$ paraformaldehyde in phosphate-buffered saline. Embryoid bodies were then embedded in OCT (Tissue-Tek) and sectioned for staining. Staining was performed by incubating for $16 \mathrm{~h}$ at $4^{\circ} \mathrm{C}$ for primary antibodies and $1 \mathrm{~h}$ at $20^{\circ} \mathrm{C}-25^{\circ} \mathrm{C}$ for secondary antibodies. After staining, samples were mounted with Fluoroshield with DAPI (Sigma). Images were acquired with a SP5 Leica confocal microscope. We used antibodies to Isl1/2 (from Developmental Studies Hybridoma Bank) and Foxpl (a gift from J. Dasen, New York University). Alexa 488 (A-11029 and A-11015) and Alexa 568 (A-11036) secondary antibodies were used (Life Technologies, 1:2000).

\section{Accession numbers}

All sequencing data have been deposited to the Gene Expression Omnibus as GSE83124.

\section{Acknowledgments}

We thank Dr. Lynne Vales, Dr. Jane Skok, Dr. Jeremy Dasen, Dr. Shengjiang Tu, Dr. Alejandro Rojas, Dr. Pedro Rocha, Dr. Ricardo SaldañaMeyer, Dr. Deneen Wellik, Dr. Cynthia Loomis, and members of the Reinberg and Mazzoni laboratories for thoughtful discussions and revision of the manuscript. We also thank Dr. Hynek Wichterle for helping to construct the iHoxc6 line, Deborah Hernandez for help with mice, the New York University Genome Technology Center for help with sequencing, and the New York University Rodent Genetic Engineering Core for help with generating the mice. This work was supported by the Howard Hughes Medical Institute and grants from the National Institutes of Health (GM-64844 and CA-199652 to D.R., T32 GM007238 to V.N., HG003143 to J.D., and R01HD079682 to E.O.M.). 


\section{References}

Beuchle D, Struhl G, Muller J. 2001. Polycomb group proteins and heritable silencing of Drosophila Hox genes. Development 128: 993-1004.

Crane E, Bian Q, McCord RP, Lajoie BR, Wheeler BS, Ralston EJ, Uzawa S, Dekker J, Meyer BJ. 2015. Condensin-driven remodelling of X chromosome topology during dosage compensation. Nature 523: 240-244.

Dasen JS, Liu JP, Jessell TM. 2003. Motor neuron columnar fate imposed by sequential phases of Hox-c activity. Nature 425: 926-933.

Dasen JS, De Camilli A, Wang B, Tucker PW, Jessell TM. 2008. Hox repertoires for motor neuron diversity and connectivity gated by a single accessory factor, FoxP1. Cell 134: 304-316.

Dekker J, Mirny L. 2016. The 3D genome as moderator of chromosomal communication. Cell 164: 1110-1121.

Dekker J, Marti-Renom MA, Mirny LA. 2013. Exploring the three-dimensional organization of genomes: interpreting chromatin interaction data. Nat Rev Genet 14: 390-403.

Dixon JR, Selvaraj S, Yue F, Kim A, Li Y, Shen Y, Hu M, Liu JS, Ren B. 2012. Topological domains in mammalian genomes identified by analysis of chromatin interactions. Nature 485: 376-380.

Dostie J, Dekker J. 2007. Mapping networks of physical interactions between genomic elements using 5C technology. Nat Protoc 2: 988-1002.

Duboule D. 2007. The rise and fall of Hox gene clusters. Development 134: 2549-2560.

Garcia-Gasca A, Spyropoulos DD. 2000. Differential mammary morphogenesis along the anteroposterior axis in Hoxc6 gene targeted mice. Dev Dyn 219: 261-276.

Guo Y, Monahan K, Wu H, Gertz J, Varley KE, Li W, Myers RM, Maniatis T, Wu Q. 2012. CTCF/cohesin-mediated DNA looping is required for protocadherin a promoter choice. Proc Natl Acad Sci 109: 21081-21086.

Guo Y, Xu Q, Canzio D, Shou J, Li J, Gorkin DU, Jung I, Wu H, Zhai Y, Tang $\mathrm{Y}$, et al. 2015. CRISPR inversion of CTCF sites alters genome topology and enhancer/promoter function. Cell 162: 900-910.

Hnisz D, Weintraub AS, Day DS, Valton AL, Bak RO, Li CH, Goldmann J, Lajoie BR, Fan ZP, Sigova AA, et al. 2016. Activation of proto-oncogenes by disruption of chromosome neighborhoods. Science 351: 1454-1458.

Lacombe J, Hanley O, Jung H, Philippidou P, Surmeli G, Grinstein J, Dasen JS. 2013. Genetic and functional modularity of Hox activities in the specification of limb-innervating motor neurons. PLoS Genet 9: e1003184.

Lonfat N, Duboule D. 2015. Structure, function and evolution of topologically associating domains (TADs) at HOX loci. FEBS Lett 589: 2869-2876.

Lonfat N, Montavon T, Darbellay F, Gitto S, Duboule D. 2014. Convergent evolution of complex regulatory landscapes and pleiotropy at Hox loci. Science 346: 1004-1006.
Lupiáñez DGG, Kraft K, Heinrich V, Krawitz P, Brancati F, Klopocki E, Horn D, Kayserili H, Opitz JM, Laxova R, et al. 2015. Disruptions of topological chromatin domains cause pathogenic rewiring of gene-enhancer interactions. Cell 161: 1012-1025.

Maeda RK, Karch F. 2009. The bithorax complex of Drosophila: an exceptional Hox cluster. Curr Topics Dev Biol 88: 1-33.

Mazzoni EO, Mahony S, Pelito M, Patel T, Thornton SR, McCuine S, Reeder C, Boyer LA, Young RA, Gifford DK, et al. 2013. Saltatory remodeling of Hox chromatin in response to rostrocaudal patterning signals. Nat Neurosci 16: 1191-1198.

McIntyre DC, Rakshit S, Yallowitz AR, Loken L, Jeannotte L, Capecchi MR, Wellik DM. 2007. Hox patterning of the vertebrate rib cage. Development 134: 2981-2989.

Montavon T, Soshnikova N, Mascrez B, Joye E, Thevenet L, Splinter E, de Laat W, Spitz F, Duboule D. 2011. A regulatory archipelago controls Hox genes transcription in digits. Cell 147: 1132-1145.

Narendra V, Rocha PP, An D, Raviram R, Skok JA, Mazzoni EO, Reinberg D. 2015. CTCF establishes discrete functional chromatin domains at the Hox clusters during differentiation. Science 347: 1017-1021.

Neijts R, Amin S, van Rooijen C, Tan S, Creyghton MP, de Laat W, Deschamps J. 2016. Polarized regulatory landscape and Wnt responsiveness underlie Hox activation in embryos. Genes Dev 30: 1937-1942.

Nora EP, Lajoie BR, Schulz EG, Giorgetti L, Okamoto I, Servant N, Piolot T, van Berkum NL, Meisig J, Sedat J, et al. 2012. Spatial partitioning of the regulatory landscape of the X-inactivation centre. Nature 485: 381-385.

Rao SS, Huntley MH, Durand NC, Stamenova EK, Bochkov ID, Robinson JT, Sanborn AL, Machol I, Omer AD, Lander ES, et al. 2014. A 3D map of the human genome at kilobase resolution reveals principles of chromatin looping. Cell 159: 1665-1680.

Rousso DL, Gaber ZB, Wellik D, Morrisey EE, Novitch BG. 2008. Coordinated actions of the forkhead protein Foxpl and Hox proteins in the columnar organization of spinal motor neurons. Neuron 59: 226-240.

Tang Z, Luo OJ, Li X, Zheng M, Zhu JJ, Szalaj P, Trzaskoma P, Magalska A, Wlodarczyk J, Ruszczycki B, et al. 2015. CTCF-mediated human 3D genome architecture reveals chromatin topology for transcription. Cell 163: 1611-1627.

Vieux-Rochas M, Fabre PJ, Leleu M, Duboule D, Noordermeer D. 2015. Clustering of mammalian Hox genes with other H3K27me3 targets within an active nuclear domain. Proc Natl Acad Sci 112: 4672-4677.

Wichterle H, Lieberam I, Porter JA, Jessell TM. 2002. Directed differentiation of embryonic stem cells into motor neurons. Cell 110: 385-397.

Yang H, Wang H, Jaenisch R. 2014. Generating genetically modified mice using CRISPR/Cas-mediated genome engineering. Nat Protoc 9: 1956-1968. 


\section{CORRIGENDUM}

Genes \& Development 30: 2657-2662 (2016)

\section{Corrigendum: CTCF-mediated topological boundaries during development foster appropriate gene regulation}

Varun Narendra, Milia Bulajić, Job Dekker, Esteban O. Mazzoni, and Danny Reinberg

In the above-mentioned article, the funding source CA-199652 to Danny Reinberg was missing from Acknowledgments. The last sentence of Acknowledgements has been corrected online to read as follows: "This work was supported by the Howard Hughes Medical Institute and grants from the National Institutes of Health (GM-64844 and CA-199652 to D.R., T32 GM007238 to V.N., HG003143 to J.D., and R01HD079682 to E.O.M.)." The authors apologize for this oversight.

doi: $10.1101 / \operatorname{gad} .306829 .117$ 


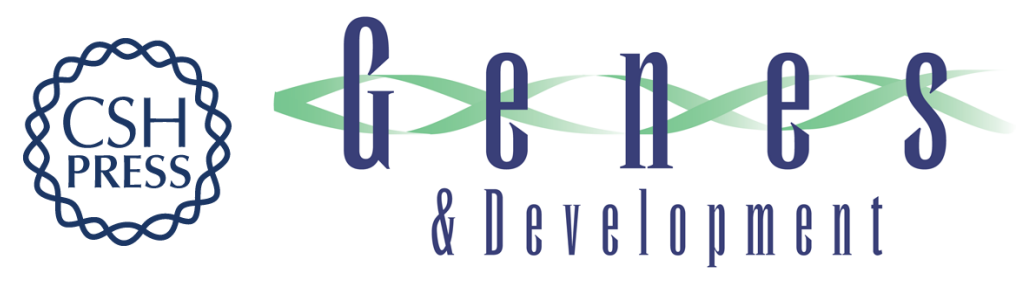

\section{CTCF-mediated topological boundaries during development foster appropriate gene regulation}

Varun Narendra, Milica Bulajic, Job Dekker, et al.

Genes Dev. 2016, 30:

Access the most recent version at doi:10.1101/gad.288324.116

\section{Supplemental http://genesdev.cshlp.org/content/suppl/2017/01/04/gad.288324.116.DC1 \\ Material}

Related Content

Corrigendum: CTCF-mediated topological boundaries during development foster appropriate gene regulation

Varun Narendra, Milia Bulajic, Job Dekker, et al.

Genes Dev. August , 2017 31: 1714

References This article cites 30 articles, 9 of which can be accessed free at:

http://genesdev.cshlp.org/content/30/24/2657.full.html\#ref-list-1

Articles cited in:

http://genesdev.cshlp.org/content/30/24/2657.full.html\#related-urls

Creative This article is distributed exclusively by Cold Spring Harbor Laboratory Press for the first Commons six months after the full-issue publication date (see

License http://genesdev.cshlp.org/site/misc/terms.xhtml). After six months, it is available under a Creative Commons License (Attribution-NonCommercial 4.0 International), as described at http://creativecommons.org/licenses/by-nc/4.0/.

Email Alerting Receive free email alerts when new articles cite this article - sign up in the box at the top Service right corner of the article or click here.

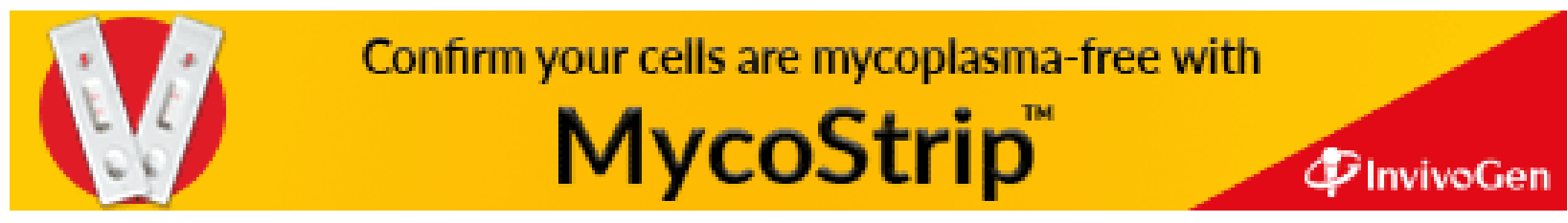

